\title{
A new (2+1)D cluster finding algorithm based on photometric redshifts: large scale structure in the Chandra deep field south
}

\author{
D. Trevese ${ }^{1}$, M. Castellano ${ }^{1}$, A. Fontana ${ }^{2}$, and E. Giallongo ${ }^{2}$
}

\author{
1 Dipartimento di Fisica, Universitá di Roma "La Sapienza”, P.le A. Moro 2, 00185 Roma, Italy \\ e-mail: dario.trevese@roma1.infn.it \\ 2 INAF - Osservatorio Astronomico di Roma, via di Frascati 33, 00040 Monte Porzio Catone, Italy
}

Received 7 April 2006 / Accepted 24 November 2006

\section{ABSTRACT}

\begin{abstract}
Aims. We study galaxy clustering and explore the dependence of galaxy properties on the the environment up to a redshift $z \sim 1$, on the basis of a deep multi-band survey in the Chandra Deep Field South.

Methods. We have developed a new method which combines galaxy angular positions and photometric redshifts to estimate the local galaxy number-density. This allows both the detection of overdensities in the galaxy distribution and the study of the properties of the galaxy population as a function of the environmental density.

Results. We detect two moderate overdensities at $z \sim 0.7$ and $z \sim 1$ previously identified spectroscopically. We find that the fraction of red galaxies within each structure increases with volume density, extending to $z \sim 1$ previous results. We measure "red sequence" slopes consistent with the values found in X-ray selected clusters, supporting the notion that the mass-metallicity relation hold constant up to $z \sim 1$.

Conclusions. Our method based on photometric redshifts allows to extend structure detection and density estimates up to the limits of photometric surveys, i.e. considerably deeper than spectroscopic surveys. Since X-ray cluster detection at high redshift is presently limited to massive relaxed structures, galaxy volume density based on photometric redshift appears as a valuable tool in the study of galaxy evolution.
\end{abstract}

Key words. galaxies: clusters: general - galaxies: evolution - galaxies: formation - galaxies: distances and redshifts cosmology: large-scale structure of Universe

\section{Introduction}

In recent years deep multi-band galaxy surveys, together with the development of reliable population syntheses, and models for galaxy formation/evolution which incorporate feedback from the galactic environment, have determined a dramatic increase of our understanding of the observed average evolution of the galaxy population in cosmic time. On the other end, clusters and groups of galaxies are the ideal laboratories where it is possible to study the environmental effects, which cause the differences of the distribution of galactic types from the average (Carlberg et al. 2001; Gomez et al. 2003). For this reason finding overdense regions at high redshift plays a central role in understanding galaxy formation and evolution. Unbiased samples of clusters and groups of galaxies can be obtained from 3D spectroscopic surveys, where the ability of a Friend of Friend (FOF) method (Huchra \& Geller 1982) to find over-densities is only limited by the uncertainty caused by the galaxy velocity dispersion. However, spectroscopic surveys reach limits of about 5 mag brighter than photometric ones, as e.g. in the SDSS survey (York et al. 2000), so that complete spectroscopic galaxy redshift surveys of large areas of the sky are not available at the boundary of the visible Universe. To minimize the effect of the background/foreground objects in the detection of galaxy overdensities, two-dimensional (2D) surveys, which are deeper, require additional a priori assumptions on either galaxy luminosity function (LF), as in the Matched Filter algorithm (Postman et al. 1996) or the presence of a red sequence (Gladders et al. 1998). Biases produced by these assumptions can hardly be evaluated for at high redshift. Multi-band photometric surveys are able to provide redshifts for large statistical samples of galaxies at the deepest observational limits, though with a lower accuracy $\left(\sigma_{z} \approx 0.05\right)$ as compared with spectroscopic ones $\left(\sigma_{z} \approx 0.001\right)$ (Fontana et al. 2000; Bell et al. 2004). Various algorithms exist to evaluate photometric redshifts. They can rely on the position in a multi-dimensional colour space respect to galaxies of known redshift (Connolly et al. 1995). Alternatively it is possible to fit the observed SED with either empirical (Lanzetta et al. 1996) or theoretical templates (Giallongo et al. 1998). Photometric redshifts have been successfully used to trace the evolution in cosmic time of the $a v$ erage galaxy population (Poli et al. 2003; Giallongo et al. 2005). However, the present accuracy of photometric redshifts leaves no room for standard FOF methods. In fact, the linking distance $\delta_{\mathrm{FOF}}$ must be larger than the distance uncertainty $\delta_{\text {phot }}$ associated with the photometric redshift, otherwise even close neighbours, which on average appear at a rms distance of the order of $\delta_{\text {phot }}$, fail to be linked. On the other hand, $\delta_{\text {FOF }}$ must be smaller than the average galaxy-galaxy distance $d_{\mathrm{g}}$, to avoid a percolation of the "friendship" path through the galaxy distribution leading to huge unphysical chains of galaxies linked by the FOF algorithm, even in the absence of any group or cluster structure: thus the condition $\delta_{\text {phot }}<\delta_{\text {FOF }}<d_{\mathrm{g}}$ must hold. However, the minimum uncertainty attained by photometric redshifts is $\Delta z \approx 0.05 \cdot(1+z)$, corresponding to $\delta_{\text {phot }} \geq$ $180 \mathrm{Mpc}$ for $z \geq 1$, while the average number density of bright galaxies is $\sim 10^{-2} \mathrm{Mpc}^{-3}$, corresponding to an average 
galaxy-galaxy distance as small as $d \approx 5$ Mpc (Bahcall 1988), i.e. we have $\delta_{\text {phot }} \gg d_{\mathrm{g}}$. This has been discussed in detail by Botzler et al. (2004) who proposed an extended friends-offriends method (EXT-FOF) which applies a FOF method to angular distances, in redshift slices which are defined on the basis of photometric redshifts, taking into account their intrinsic uncertainties. In the present paper we present an alternative approach to the use of photometric redshifts. Our algorithm evaluates the 3D galaxy density using angular positions and photometric redshifts with the purpose of: i) detecting galaxy overdensities in three dimensions; and ii) assigning to each galaxy a measure of the environmental density, to extend the analysis of the environmental effects on galaxy evolution to the limits of photometric surveys. The method is applied to a deep photometric survey of the Chandra Deep Field South (Cimatti et al. 2002a), where we re-discover some previously known overdensities and we find a clear relation between the fraction of blue and red galactic types and the local density. The paper is organised as follows. In Sect. 2 we present a new method to define a galaxy-volume density using angular positions and photometric redshifts. In Sect. 3 we apply the algorithm to a deep photometric survey of a portion of the Chandra Deep Field South to identify some high- $z$ clusters and analyse the dependence of their galaxy population on local density. In Sect. 4 we study the evolution in cosmic time of the red sequence. In Sect. 5 we check the reliability of the results and possible extrapolation to deeper surveys by applying the algorithm to a simulated galaxy catalogue. In Sect. 6 we discuss the results and Sect. 7 provides a summary. Throughout the paper we adopt consensus cosmological parameters $\Omega_{\Lambda}=0.7, \Omega_{\mathrm{M}}=0.3, H_{0}=70 \mathrm{~km} \mathrm{~s}^{-1} \mathrm{Mpc}^{-1}$.

\section{2. (2+1)-dimensional density from photometric redshifts}

As far as background/foreground effects are acceptable, namely at relatively low redshifts, the surface density is sufficient to detect galaxy clusters or to study their morphology, as in the case of Abell (1958) or Zwicky (Zwicky et al. 1961) clusters or in the later works of the Edinburgh-Durham Cluster Catalogue (Lumsden et al. 1992). A surface density

$\Sigma_{n}=n /\left(\pi D_{\mathrm{p}, n}^{2}\right)$

of galaxies can be computed considering the projected distances $D_{\mathrm{p}, n}$ to the $n$th nearest neighbour, and can be used to study the effect of the environment on the distribution of galactic types, as done by Dressler (Dressler 1980; Dressler et al. 1997). Similarly, when spectroscopic redshifts are available, it is possible to compute the three-dimensional (non projected) distance $D_{n}$ to the $n$th nearest neighbour and derive a volume density

$\rho_{n}=3 n /\left(4 \pi D_{n}^{3}\right)$.

The method we propose for deep photometric surveys, where spectroscopic galaxy redshifts are not available, consists in combining, in the most effective way, the angular position with the (much less accurate) distance as computed from the photometric redshift. In principle it is possible to compute for each galaxy the distance from its neighbours from the angular positions and photometric redshifts, once a cosmological model is assumed. In practise we prefer to divide the survey volume in cells whose extension in different directions $(\Delta \alpha, \Delta \delta, \Delta z)$ depends on the relevant positional accuracy and thus are elongated in the radial direction. Then for each point in space (i.e. for each cell) we count neighbouring objects of increasing distance, until a number $n$ of objects is reached. We define the density associated to the cell as

$\rho=n / V_{n}$

where $V_{n}$ is the volume which includes the $n$ nearest neighbours. The choice of $n$ is a trade off between spatial resolution and signal-to-noise ratio. In the following the number $n$ is chosen in such a way that $n$ objects are present in a single cell in the regions of maximum density. Notice that, in principle, the way of searching neighbouring objects of increasing distance is not univocal, since distance steps (cell sizes) in different directions can be chosen arbitrarily. The result will be a different smoothing, and resolution, in different directions. The choice we adopt is to keep the maximum resolution in transversal and radial directions allowed by the data. Since the uncertainty in the radial direction is much larger, this will correspond to elongated cells and lower radial resolution. Steps $\Delta z$ in the radial direction smaller than the photometric redshift uncertainty would uselessly increase the computing time. In counting galaxies we must take into account the increase of limiting luminosity with increasing redshift for a given flux limit. If $m_{\text {lim }}$ is the limiting (apparent) magnitude in a fixed observing band, at each redshift $z$ we detect only objects brighter than an absolute magnitude $M_{\text {lim }}(z)$, decreasing (brightening) with $z$. We can assume a reference redshift $z_{\mathrm{c}}$ below which we detect all objects brighter than the relevant $M_{\mathrm{c}} \equiv M_{\mathrm{lim}}\left(z_{\mathrm{c}}\right)$, below which we neglect the incompleteness. At $z>z_{\mathrm{c}}$ the fraction of detected objects is:

$s(z)=\frac{\int_{-\infty}^{M_{\lim (z)}} \Phi(M) \mathrm{d} M}{\int_{-\infty}^{M_{\mathrm{c}}} \Phi(M) \mathrm{d} M}$

where $\Phi(M)$ is the galaxy luminosity function. Thus, in evaluating the galaxies number density, we apply a limiting magnitude correction by assigning a weight $w(z)=1 / s(z)$ to each detected galaxy of redshift $z$. In this way we correct the systematic underestimate of density, caused to the increasing fraction of galaxies which fall below the brightness limit for increasing redshift. Of course the noise in the derived density increases as the square root of $w(z)$, but the advantage is to obtain a density scale independent of redshift, at least to a first approximation. We stress that, in general, the distance modulus $m-M$ depends not only on the luminosity distance $D_{\mathrm{L}}(z)$, and thus on the adopted cosmological model, but also on the $k$ - and evolutionary-corrections which, in turn, depend on the galactic type. Moreover the luminosity function itself depends on both the wavelength $\lambda$ and cosmic time. However, as far as $s(z)$ is not much smaller than one, it is possible to adopt simple representations of these effects, and any correction to the limiting magnitude correction will represent a second order effect. Practically the choice of the cell sizes is determined by the accuracy of the photometric redshifts. In fact, once the cells have transversal sizes which are much smaller than the radial one (say 1/1000), a further increase of the transversal resolution does not justify the corresponding increase of the computing time. At least, this is true as far as we do not expect structures which are physically strongly elongated in the radial direction. In the application presented in the following sections, the relative redshift uncertainty is $\Delta=\left|z_{\text {spec }}-z_{\text {phot }}\right| /(1+z) \approx 0.05$ (Cimatti et al. 2002b), corresponding to a length scale $l_{z} \approx 200$, Mpc at $z \sim 1$. Cells sizes are arbitrary and are chosen small enough not to degrade the spatial resolution, while avoiding a useless increase of the computing time. We have adopted $\sim 60 \mathrm{Mpc}$ in the radial direction and $\sim 60 \mathrm{kpc}$ at $z \sim 1$ in transversal direction, corresponding 


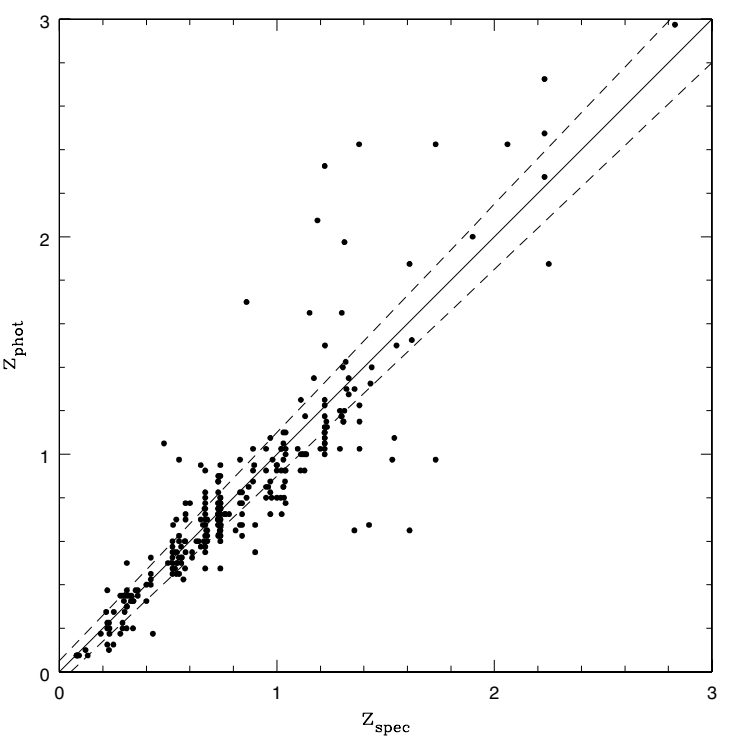

Fig. 1. Photometric redshifts $z_{\text {phot }}$ versus spectroscopic redshifts $z_{\text {spec }}$ for all the galaxies in the catalogue with spectroscopic observations (Grazian et al. 2006, and refs. therein). Dashed lines indicate the rms uncertainty $0.05(1+z)$.

to $\Delta \alpha=\Delta \delta=3.68$ arcsec. Galaxy clusters or, more generally, galaxy overdensities are defined as connected 3-dimensional regions with density exceeding a fixed threshold. Once overdensities are identified, it is possible to analyse the properties of galaxies as a function of the local density.

\section{Galaxy clusters in the Chandra deep field south}

The dataset used to identify distant clusters and to study the properties of their member galaxies is the deep photometric catalogue of the K20 survey (Cimatti et al. 2002a,b) containing photometry in the UBVRIZJK bands of a $6.38 \times 6.13$ arcmin field in Chandra Deep Field South (CDFS) (Giacconi et al. 2002). The sample is limited to $I_{\mathrm{AB}}<25$, while the photometric depth in the other bands allows to assume that virtually all galaxies in the catalogue have 8-band photometry, except a few objects with very extreme colours. We have added to the spectroscopic redshifts of the K20 all public spectroscopic redshifts in our field from GOODS-MUSIC catalogue (Grazian et al. 2006, and refs. therein). The catalogue contains 1749 galaxies among which 292 have spectroscopic redshifts and the remaining have only photometric redshifts. The procedure for deriving photometric redshifts and test their accuracy is described in Cimatti et al. (2002b), where it is shown that the distribution of the fractional error $\Delta \equiv\left(z_{\text {spec }}-z_{\text {phot }}\right) /\left(1+z_{\text {spec }}\right)$ is not Gaussian. After excluding "outlayers" with $\Delta>0.15$, which represent less than $9 \%$ of the total, we obtain $\sigma_{\Delta}=0.05$.

Figure 1 shows photometric redshifts versus spectroscopic redshifts, with the uncertainty $0.05(1+z)$ indicated by the dashed lines.

We have constructed $(2+1) \mathrm{D}$ maps of the volume density $\rho_{n}$, with $n=10$, computing $s(z)$ in Eq. (3) on the basis of a cosmologically evolving luminosity function. This has been taken from Poli et al. (2003), who derived the rest-frame $B$ luminosity function which is directly sampled until the rest-frame blue is observed in the $K$ band, namely up to a redshift of about 3.5. In their analysis Poli et al. (2003) find little density evolution at the faint end with respect to the local values, while at the bright end
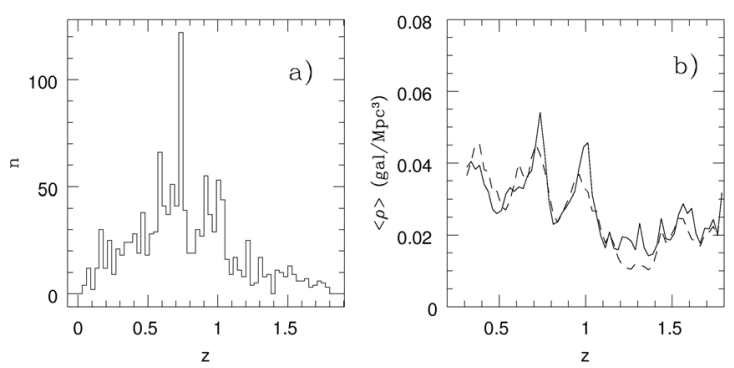

Fig. 2. a) The distribution of photometric redshifts of the sample. b) Average $\rho_{10}$ density on the entire field, in redshift bins, versus $z$ as determined by the $(2 \mathrm{D}+1)$ algorithm using for all sources: i) photometric redshifts (dashed line); ii) photometric redshifts, or spectroscopic redshifts whenever available (continuous line).

a brightening increasing with redshift is apparent with respect to the local LF.

The choice $n=10$ corresponds to the maximum number of objects in a single cell at high density as discussed in Sect. 2.

To see how the resolution of photometric redshifts compares with real objects distribution, we show in Fig. 2 the histogram of photometric redshifts in the field. Two main clumps appear about redshifts $0.70,1.00$. A comparison with the distribution of spectroscopic redshifts of the CDFS (see Gilli et al. 2003, Fig. 1) clearly indicates the reality of the two clumps, although the two peaks at $z=0.67$ and $z=0.73$ found by Gilli et al. (2003) in the distribution of spectroscopic redshifts are barely resolved in our photometric redshift distribution. On the basis of spectroscopic redshifts, Gilli et al. (2003) found that both structures are spread over the field, although the latter includes a cD galaxy, suggesting a dynamically relaxed status (see Sarazin 1988).

To check to what extent our results depend on the presence of spectroscopic redshift in our catalogue, we show in Fig. $2 b$ the average density in the field as a function of redshift, as computed from photometric redshifts only, or including also spectroscopic redshifts whenever the latter are available. The two curves look similar. In fact using $z_{\text {spec }}$ instead of $z_{\text {phot }}$ does not change significantly the density, once the cell size has been chosen on the basis of the (much lower) $z_{\text {phot }}$ accuracy. This means that our results do not rely on the availability of several spectroscopic redshifts in the field. Notice that the density reported in Fig. 2b, which is averaged over the entire field, is not used to detect structures. For this purpose we select individual cells with density above a given threshold, and then we look for connected volumes. The choice of the threshold is an arbitrary trade off between completeness and reliability. From our numerical simulations (see Sect. 5) we found that a threshold corresponding to about three to five times the average density can identify richness zero Abell clusters. More complex simulations would be necessary to reliably evaluate the degree of contamination as a function of the richness class and redshift. In real data, from the distribution of the densities of individual cells we found that thresholds of $0.078 \mathrm{Mpc}^{-3}$ or $0.13 \mathrm{Mpc}^{-3}$ (i.e. 3 or 5 times the average) isolate $2.2 \%$ or $0.5 \%$ respectively of the total cell number. To avoid excessive contamination from random density fluctuations we adopted a threshold $\rho_{10}^{\text {thresh }}=0.08 \mathrm{Mpc}^{-3}$ which selects about $2.0 \%$ of the cells. In this way we identified three over-densities listed in Table 1. We adopted a redshift independent threshold to detect structures of comparable density at any redshift. In principle, this implies an higher probability of contamination at higher redshifts: we plan to explore this issue in future work extending to higher redhifts. 
Table 1. Detected structures.

\begin{tabular}{cccc}
\hline \hline$\#$ & $\alpha_{2000}$ & $\delta_{2000}$ & $z$ \\
\hline 1 & 033220.08 & -274707.20 & 0.70 \\
2 & 033216.74 & -274552.99 & 1.00 \\
3 & 033216.51 & -274645.95 & 1.55 \\
\hline
\end{tabular}

The first structure, at $z=0.70$ is approximately centred on the cD galaxy, whose position, in turn, corresponds to the centre of the extended X-ray source CDFS566 (Giacconi et al. 2002). As noted above, we cannot resolve, in redshift space, the walllike structure at $z=0.67$ described by Gilli et al. (2003) which then contaminates the structure at $z=0.70$. In spite of that we find a relatively concentrated structure with full width at half maximum of about $0.12 \mathrm{Mpc}$. After the statistical subtraction of the background/foreground field galaxies the number of galaxies within the Abell radius $R_{\mathrm{A}}$ is $N_{\mathrm{c}}=182$, of which 38 are between $m_{3}$ and $m_{3}+2$, corresponding to a richness class 0 . From the spectroscopic redshifts we can evaluate a velocity dispersion along the line of sight, $\sigma_{\mathrm{p}}=334 \pm 31 \mathrm{~km} \mathrm{~s}^{-1}$, for the 39 galaxies belonging to the peak in the redshift distribution centred at at $z=0.73$, the uncertainty being computed by a bootstrap method. The relevant virial mass $M=\frac{3 \pi}{2} \frac{\sigma_{\mathrm{P}}^{2} R_{\mathrm{PV}}}{G}$ is $M=1.19 \times$ $10^{14} M_{\odot}$, where $R_{\mathrm{PV}}=N(N-1) / \sum_{i>j} R_{i j}^{-1}$ is the projected virial radius and $R_{i j}$ are the projected distances between each pair of the $N=39$ galaxies (Heisler et al. 1985; Girardi et al. 1998). For the sole purpose of displaying the morphology of the density field we show, in Fig. 3a, the isolines of the surface density $\Sigma_{10}$ (see Sect. 2), evaluated in the redshift slice $0.70<z_{\text {phot }}<$ 0.75 . A similar plot for the overdensity at $z \simeq 1.00$ is shown in Fig. $3 \mathrm{~b}$ where the galaxy number within the Abell radius, with $m_{3}<m<m_{3}+2$, barely reaches the formal threshold of 30 corresponding to richness class 0 , depending on the exact location of the adopted centre. The spectroscopic redshift distribution suggests the existence of two distinct peaks at 0.97 and 1.04: the former associated with galaxies around the main overdensity and the latter corresponding to the south east extension. The analysis of possible substructures of this overdensity requires further spectroscopic data. The third clump we find at $z=1.55$ does not appear in the distribution of spectroscopic galaxy redshifts which is limited to brighter fluxes respect to our photometric data. On the other hand, the accuracy of our photometric redshifts is statistically checked against spectroscopic ones only for $z_{\text {spec }} \lesssim 1$, so that further data would be needed to assess the reality of this structure. However a peak in the distribution of the $\mathrm{X}$-ray selected AGNs in the field is present at about $z=1.55$. Thus, as far as we can assume that distribution of AGNs traces the large scale distribution of matter we can say that we are detecting a structure not previously seen in galaxy spectroscopic surveys. The Abell richness of the third structure at $z \sim 1.55$ cannot be evaluated, since $m_{3}+2$ falls below the limiting magnitude $m_{I}=25$. The peak in the photometric redshift distribution contains 57 objects spread along a moderate over-density crossing the field from north-west to south-east likely related to the above discussed large-scale structure traced by X-ray selected AGN (Gilli et al. 2003). The association of an environmental density with individual galaxies allows both a further assessment of the nature of the detected overdensities and the analysis of the relation between galaxy spectral energy distribution and the environment.

A strong colour bi-modality of the colour distribution has recently been confirmed on the basis of a large galaxy sample of about 150000 objects from the Sloan Digital Sky Survey

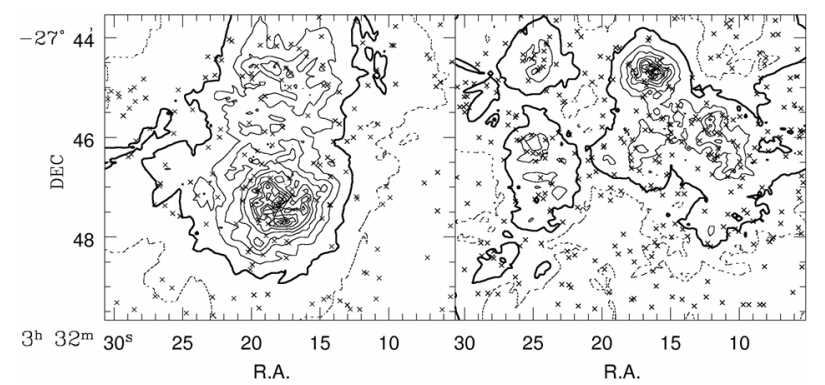

Fig. 3. Isodensity contours of the surface density $\Sigma_{10}$ as computed in the redshift slice $0.70<z<0.75$, which includes the first detected structure (left panel). b) Same plot for the second structure in the redshift slice $0.90<z<1.10$ (right panel).

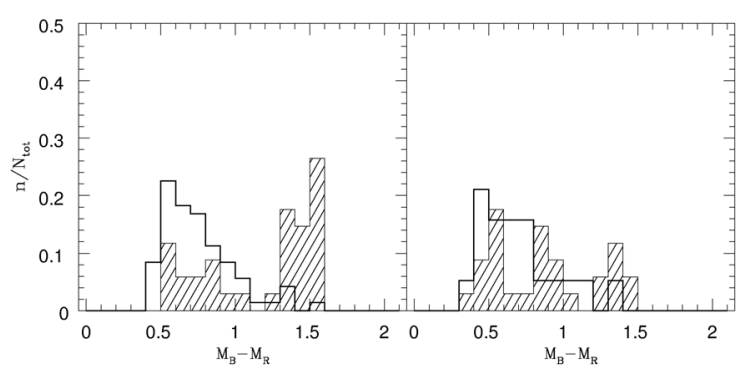

Fig. 4. Galaxy colour distributions: at high density $\left(\rho_{10}>0.08 \mathrm{Mpc}^{-3}\right)$ (shaded histogram), at low density $\left(\rho_{10}<0.03 \mathrm{Mpc}^{-3}\right)$, for the two structures at $z \sim 0.70$ (left) and $z \sim 1.0$ (right).

(Strateva et al. 2001, and refs. therein). This bi-modality has been shown to maintain up to $z \approx 2-3$ (Giallongo et al. 2005), with a local minimum in the colour distribution which evolves in redshift and represents the natural separation between the "blue" and "red" galaxy populations, the latter defining an $a v$ erage red sequence of the field. Instead, Fig. 4 shows rest-frame $B-R$ colour distribution in overdense $\left(\rho_{10}>0.08 \mathrm{Mpc}^{-3}\right)$ and underdense $\left(\rho_{10}<0.03 \mathrm{Mpc}^{-3}\right)$ regions both at $z \sim 0.7$ and $z \sim 1$. Following Carlberg et al. (2001), we conservatively adopt the constant rest frame colour $B-R=1.25$ as a boundary between the two populations. The excess of red galaxies in overdense regions respect to the field, clearly appears in Fig. 4. According to a Kolmogorov-Smirnov test, the probability of the null hypothesis that the colours inside and outside the overdensities are randomly drawn from the same distribution is $4.2 \times 10^{-7}$ for the cluster at $z=0.7$ and 0.058 for the cluster at $z=1.0$. Due to the insufficient statistics, a similar colour segregation cannot be detected in the case of the $z \sim 1.55$ overdensity, which will be studied as soon as deeper photometric data will become available (Trevese et al. 2006). In the two former overdensities we can study the fraction of red galaxies as a function of the density $\rho_{10}$. The result is shown in Fig. 5, where both clusters show a decrease of red fraction as a function of $\rho_{10}$, marginally significant at $z=1.0$ and more evident at $z=0.7$. This colour segregation is clearly related to the morphological segregation first found by Dressler (1980) for local clusters successively extended to $z=0.5$ by Dressler et al. (1997). Our analysis of colour segregation allows to extend a quantitative investigation of environmental effects up to redshifts where morphological studies become unfeasible. 


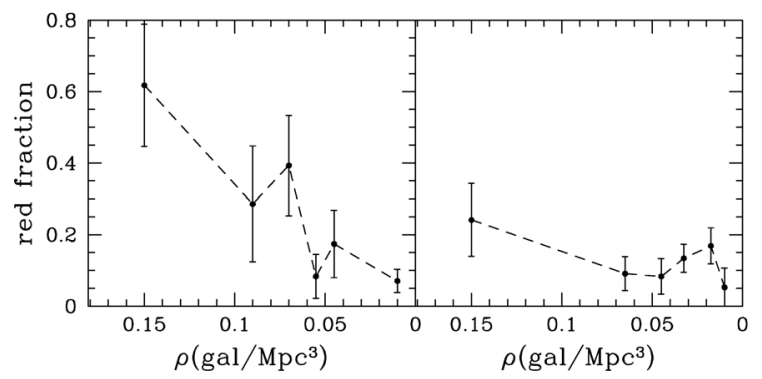

Fig. 5. Fraction of galaxies with rest frame colour $B-R>1.25$ as a function of the volume density $\rho_{10}$, for the two structures at $z \sim 0.70$ (left) and $z \sim 1.0($ right). Error bars represent Poissonian fluctuation.

\section{Cosmological evolution of the red sequence}

We first study the cosmological evolution of the average red sequence of the field. Following Bell et al. (2004) it is possible to define for each galaxy a colour index $C^{\prime}$ reduced to $M_{V_{\text {rest }}}=-20$ by shifting each galaxy in the C-M diagram to $M_{V_{\text {rest }}}=-20$ along the red sequence:

$C^{\prime}=C+\alpha_{\mathrm{RS}} \cdot\left(M_{V \text { rest }}-20\right)$

where $C$ is the rest-frame colour, $(U-V)_{\text {rest }}$ in our case, and $\alpha_{\mathrm{RS}} \equiv \partial(U-V)_{\text {rest }} / \partial M_{V_{\text {rest }}}$ is the slope of the red sequence, In practise, for a direct comparison with Bell et al. (2004) who adopt a different cosmology, we assume as reference magnitude $M_{V_{\text {rest }}}=-20.7$ instead of -20 . A distribution of the $C^{\prime}$, instead of $C$, colours allows a better identification of the "Early type", or red galaxies, population which defines the average red sequence. Following Bell et al. (2004) we assume a constant slope $\partial(U-V)_{\text {rest }} / \partial M_{V_{\text {rest }}}=-0.08$ which is derived from a sample of nearby clusters (Bower et al. 1992). This assumption is justified by the analysis of Blakeslee et al. (2003) who find a constant slope for different galaxy clusters up to $z=1.2$ (see the next paragraph). At higher redshifts Giallongo et al. (2005) find a decrease of the red sequence slope $\partial(U-V)_{\text {rest }} / \partial M_{B_{\text {rest }}}$ from -0.098 to -0.062 , in the two wide redshift bins $0.4-1$ and $1.3-3.5$ respectively. In the present analysis, which is limited to $z<1.7$, we neglect this change of slope and we estimate the red sequence colours in three relatively narrow redshift bins. Figures $6 a-c$ shows the rest-frame colour-magnitude diagrams for all galaxies in the field, selected in the three slices of photometric redshift, $0.7<z<0.8,0.9<z<1.1,1.4<z<1.7$ and the relevant $C^{\prime}$ distributions. From the $C^{\prime}$ colour distribution we select the members of the red population as those galaxies lying redwards of the natural minimum which separates the blue and red populations. A fit in the C-M diagram of the red population with a straight line of fixed slope $\alpha_{\mathrm{RS}}$ defines the colour $C_{\mathrm{RS}}^{\prime}$ of the average red sequence in different bins of redshift. Figure 7 , adapted from Bell et al. (2004) shows the colour $C_{\mathrm{RS}}^{\prime}$ of the $a v$ erage red sequence in our field in the above redshift intervals, compared with the colour of the average red sequence as a function of redshift obtained from COMBO17 data, after a correction on $\Delta C^{\prime}=C_{\text {combo }}^{\prime}-C_{\mathrm{CDFS}}^{\prime}=-0.08$ as derived from a comparison of the colours of those high- $z$ galaxies which are in common in the 2 catalogues COMBO17 and K20. Our error bars represent the rms dispersion of $C^{\prime}$ in each redshift bin. We can conclude that the $\Delta C^{\prime}$ vs. $z$ relation in our analysis is consistent with Bell et al. (2004) up to $z \simeq 1$, moreover the point at $z=1.5$ lies on the linear extrapolation of their points. As a further step, we measured the slope of the red sequence in the two clumps at redshifts 0.7 and 1.0. This has not be done for the third clump at $z=1.55$, due to insufficient statistics. We selected

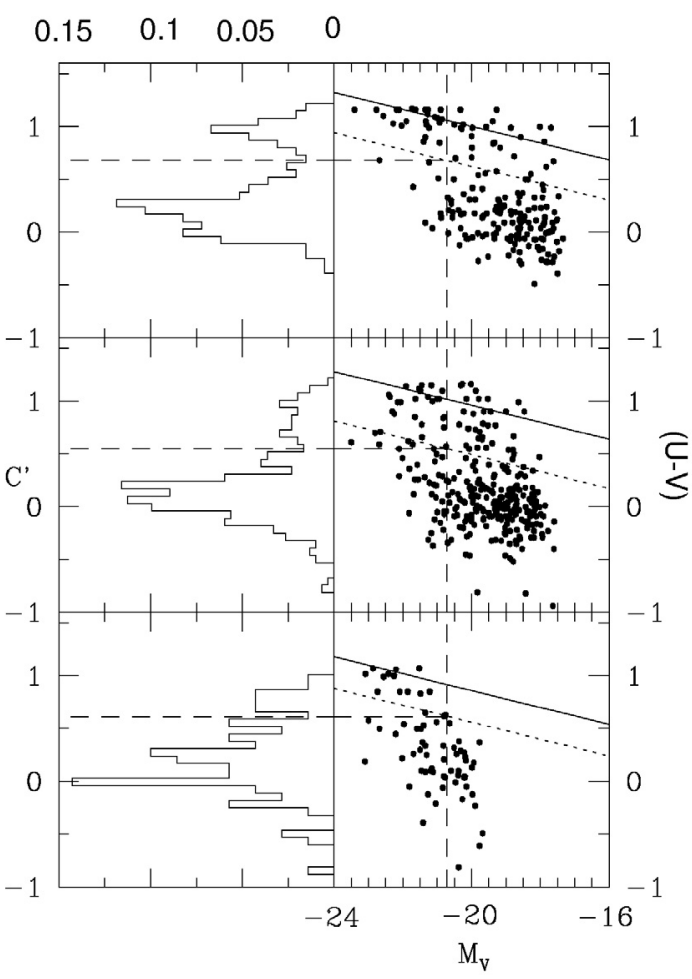

Fig. 6. Left panels: histograms of the $C^{\prime}$ colour, defined in the text, for all the objects in the intervals $0.7<z<0.8,0.9<z<1.1,1.4<z<1.7$ (from the top). Dashed lines, corresponding to a local minimum, define the red and the blue populations. Right panels: rest-frame $(U-V)$ vs. $M_{V}$ diagrams: the continuous line represents the fit to the points belonging to the red population, with a fixed slope $\alpha_{\mathrm{RS}}=0.08$. The dashed vertical line represents the reference absolute magnitude $M_{V}=-20.7$. The dotted line indicates the valley separating the two populations.

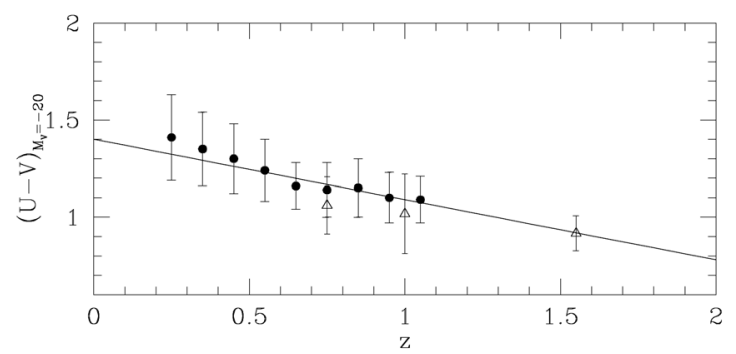

Fig. 7. The colour $C_{\mathrm{RS}}^{\prime}$ at $M_{V_{\text {rest }}}=-20$ of the average red sequence, as computed from COMBO17 survey (circles), and resulting from the present analysis (triangles). The straight line represents a linear fit on all the data analysed in the COMBO17 survey (adapted from Bell et al. 2004).

galaxies with an environmental density above a threshold $\rho_{10}=$ $0.08 \mathrm{gal} / \mathrm{Mpc}^{3}$ (see Sect. 3). We then isolated the red population from the histograms of the $C^{\prime}$ colour which are clearly bimodal. Finally, we evaluated the slope of the colour-magnitude relation for this population in the $U-B$ vs. $B$ diagram, as done by Blakeslee et al. (2003). Our results show that the slope of the red sequence is consistent with being the same in local clusters and in the two main overdensities $(z \sim 0.7$ and $z \sim 1.0)$. Blakeslee et al. (2003) found little or no evidence of evolution of this slope, out to $z=1.2$. Our results (Fig. 8) lie within $1 \sigma$ from their average value $\left|\left\langle\partial(U-B)_{\text {rest }} / \partial M_{B_{\text {rest }}}\right\rangle\right|=0.032$. According to the standard interpretation (Arimoto \& Yoshii 1987; Kauffmann \& Charlot 1998) this implies that the mass-metallicity relation holds the same from $z=0$ up to at least $z=1$. 


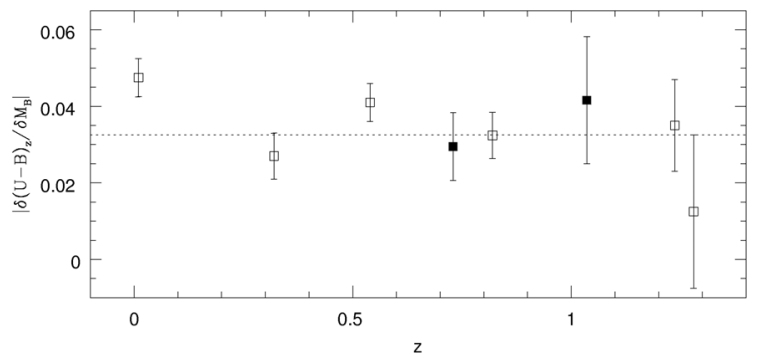

Fig. 8. The slope of the rest-frame $(U-B)$ vs. $M_{B}$ in the galaxy clusters collected by Blakeslee et al. (2003) (open squares) and in the two structures detected in the CDFS (filled squares). The dotted line represents the average value derived by Blakeslee et al. (2003).

\section{A check of the algorithm on simulated clusters}

To check the reliability of the algorithm in detecting clusters of various types and redshifts we created a series of mock catalogues including field galaxies and clusters. Field objects were uniformly distributed on a square of $6 \times 6$ arcmin centred on the cluster, with a space density and absolute magnitude distributions assigned according to the redshift-dependent Schechter-like (Schechter 1976) LF derived by Giallongo et al. (2005):

$\Phi(M, z) \mathrm{d} M=0.4 \ln 10 \phi^{*}(z)\left[10^{0.4\left(M^{*}(z)-M\right)}\right]^{1+\alpha} \exp \left[-10^{0.4\left(M^{*}(z)-M\right)}\right]$,

with $\phi^{*}=\phi_{0}^{*}(1+z)^{\gamma}$ and $M^{*}=M_{0}^{*}-\delta \log (1+z)$. Clusters are represented by a number-density distribution

$n(r)=n_{0}\left[1+\left(r / r_{\mathrm{c}}\right)^{2}\right]^{-3 / 2}$

(see Sarazin 1988) with a typical core radius $r_{\mathrm{c}}=0.25 \mathrm{Mpc}$. To take into account the uncertainty on photometric redshifts, we assigned to each galaxy a random redshift with a mean corresponding to the cluster redshift and a dispersion $\sigma_{z}=0.05$ corresponding to a velocity dispersion along the line of sight $\sigma_{v}=15000 /(1+z) \mathrm{km} \mathrm{s}^{-1}$ (Hogg 2000). Once position and redshift are assigned to a galaxy, an absolute blue magnitude $M_{B}$ is extracted from a Schechter LF, with parameters $M_{B}^{*}=-20.04$ and $\alpha=-1.05$ for ellipticals and $M_{B}^{*}=-19.48$ and $\alpha=-1.23$ for spirals, derived by De Propris et al. (2003) from the analysis of $2 \mathrm{dF}$ clusters. The fraction of different galaxy types was chosen according to Goto et al. (2004). Different values of the central density $n_{0}$ are chosen to produce clusters of various richness classes. Table 2 reports the values of the parameters for elliptical and spiral galaxies in the field. To evaluate the total number of cluster and field objects $N=\int_{-\infty}^{M_{\mathrm{LIM}}(z)} \Phi(M, z) \mathrm{d} M$ we computed the limiting rest-frame magnitude in the $B$ band given by:

$M_{\mathrm{LIM}}=m_{l}+C-K-E-25.0-5.0 \cdot \log _{10} D_{\mathrm{L}}$

$m_{l}=25$ is the limiting $A B$ magnitude at the effective wavelength of the $I$ band, $K$ and $E$ are, respectively, the type dependent $K$-correction and evolutionary correction (Poggianti 1997), $\mathrm{C}$ is the relevant rest-frame $(B-I)$ colour and $D_{\mathrm{L}}$ is the luminosity distance. We assumed a fixed proportion of $\mathrm{Sa}(70 \%)$ and Sc $(30 \%)$ in the field catalogue. Various mock catalogues were generated at each cluster redshift $0.5,1.0,1.5,2.0$ corresponding to Abell (1958) richness classes 0, 1, 2, 3. Figure 9 shows the galaxies belonging to a richness class 0 cluster, above a $\rho_{10}$ density threshold five times the average, as seen at different redshifts and limiting magnitudes. Real cluster members and interlopers are represented by filled dots and crosses respectively. For a limiting magnitude $m_{\text {lim }}=25$, the cluster is detected with an acceptable contamination up to $z=1$, while $z=1.5$ may be assumed as
Table 2. LF parameters.

\begin{tabular}{cccccc}
\hline \hline Type & $\phi_{0}^{*}$ & $\gamma$ & $M_{0}^{*}$ & $\delta$ & $\alpha$ \\
\hline Ellipticals & 0.0106 & -2.23 & -20.03 & 2.72 & -0.46 \\
Spirals & 0.0042 & -0.52 & -20.11 & 2.35 & -1.38 \\
\hline
\end{tabular}

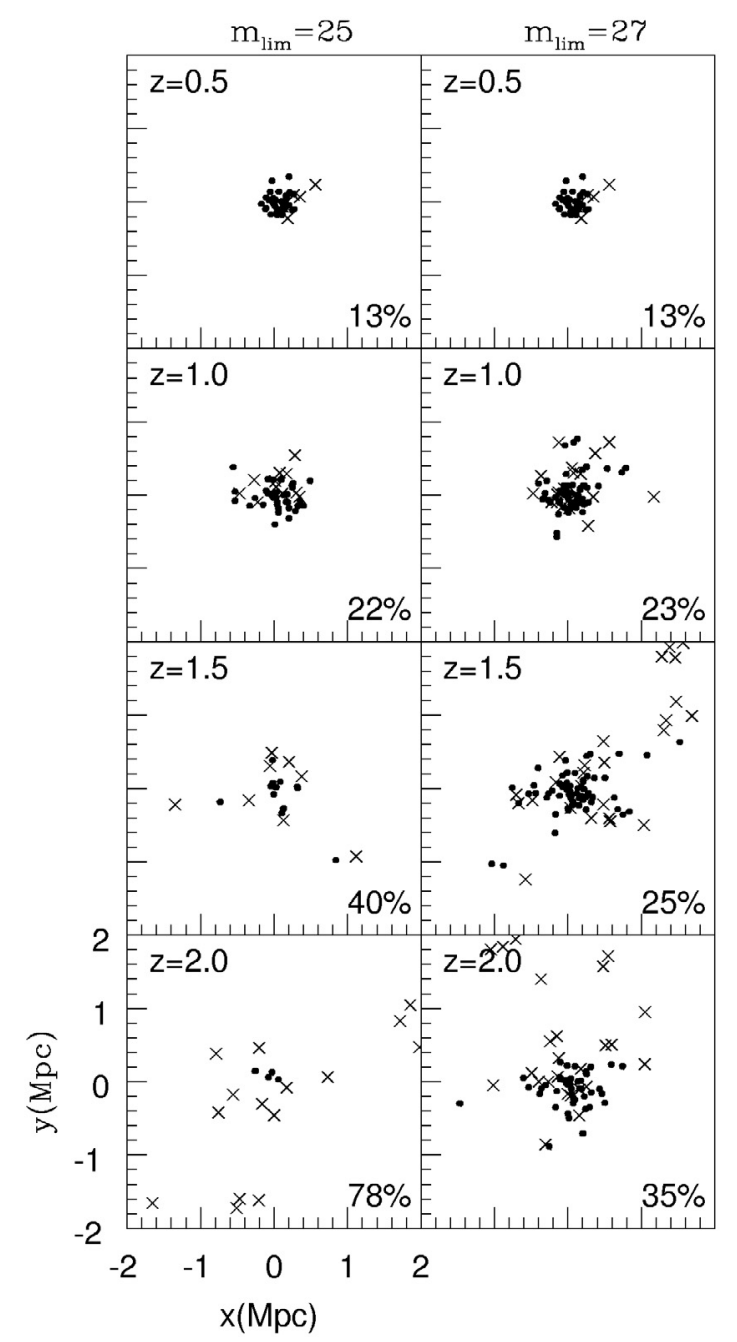

Fig. 9. Galaxies above an environmental density $\rho_{10}$ threshold 5 times the average, for redshifts $0.5,1.0,1.5$ and 2.0 and for catalogues with two different limiting magnitudes. Filled dots represent real members of the simulated richness class 0 clusters, while crosses represent interlopers. The contamination is reported in each panel.

a detection limit. However, in the case of a deeper survey, with $m_{\text {lim }}=27$ comparable with the new GOODS-MUSIC catalogue (Grazian et al. 2006), whose analysis is in progress, the same 0 richness cluster is well detected up to $z=1.5$ and still visible at $z=2$.

Clearly the ability of the algorithm in separating the overdensities increases with their richness and their angular distance. Thus, to perform a conservative evaluation of the algorithm, we simulated two relatively low density structures perfectly aligned along the line of sight. We have considered pairs of overdensities, representing two richness 0 clusters as those described above. The first is at redshift $z=1.0$ and the second at various higher redshifts. We found that, depending on the chosen threshold, the two structures can be separated if the distance is greater then $\Delta z=0.15$. However, once $N$ galaxies are assigned to a single structure, the redshift of the latter can be determined with 
an accuracy of about $\Delta z / N^{1 / 2}$, which can be an order of magnitude smaller than $\Delta z$. The above results refer to the possibility of resolving (aligned) structures. It is worth noting that in real cases, once a $(2+1) \mathrm{D}$ density map is available, it is possible to adopt a multi-threshold technique, to identify possible physical substructures and/or projection effects.

\section{Discussion}

It is clear that many methods exist for detecting clusters, depending on the type of the available data: from single-band images, where only brightness can be used to complement angular positions in the identification of overdensities like in matched filter methods (Postman et al. 1996), to images in a few bands where colours allow to identify the cluster red sequence (Gladders et al. 1998), to images in several bands allowing the determination of photometric redshifts, to spectroscopic redshift. Each method has his advantages and limitations. One of the main issues is our ability to compare the results of numerical simulations with galaxy catalogues obtained from different data sets. A general discussion of these problems has recently presented by Gal (2006). To establish whether one method is more or less efficient respect to the another in detecting structures is not a straightforward task. Given a mock catalogue, each method should be optimised, by a proper choice of the relevant parameters, to obtain a meaningful comparison. Moreover, mock catalogues obtained by different assumptions on cosmic evolution of the spectral and clustering properties of galaxies would lead to different optimisations. The problem of using the photometric redshifts to identify structures in the galaxy distribution has been tackled recently by Botzler et al. (2004) who discuss why standard FOF methods, suited for spectroscopic redshift surveys, cannot be simply applied to photometric redshift surveys without a proper account of the large inherent uncertainties. They propose an extended EXT-FOF method which applies a twodimensional FOF algorithm to slices of the galaxy catalogue, which are defined by photometric redshifts, taking into account their uncertainty. Clearly photometric redshifts add crucial information for the identification of structures in the galaxy distribution through the fitting of the observed spectral energy distribution to either theoretical or observed galaxy templates, evolving in cosmic time. Here we propose and discuss a different way of using photometric information: instead of comparing the distance between galaxy pairs, our method uses the statistical information about how many galaxies are in the neighbourhood of a given point and estimates a local density without introducing a fixed smoothing scale. A comparison of our method with EXT-FOF or other methods is beyond the aim of the present work and will be the subject of future investigations. What we want to stress here are only the new informations we obtain with our method: i) the spatial resolution is maximised in each point, since near density peaks the volume taken into account to evaluate the density is small, while it becomes large where the density is low; ii) once the density map is available, it can be analysed in different ways without re-running the algorithm. For instance the map can be sliced at different density thresholds to see how structures which appear separated at high density merge at lower densities. In principle it is also possible to apply an harmonic analysis to density maps.

We have also obtained some new results by applying our $(2+1) \mathrm{D}$ method to real data in the Chandra Deep Field South which is one of the most studied fields in the sky. The existence of deep X-ray data from the Chandra observatory provided a sample of Active Galactic Nuclei (AGN) in the field
(Giacconi et al. 2002). The spectroscopic follow up has shown the existence of large-scale structure (Gilli et al. 2003). AGN, thanks to their intrinsic luminosity and to strong emission lines, are the ideal tracers of the mass distribution at the maximum distance reachable by optical spectroscopy, under the assumption that their spatial distribution mimics that of normal galaxies. The same depth is either unreachable or requires huge exposure times for normal galaxies. Photometric redshifts, though with the limitation imposed by their poor accuracy, permit to identify overdensities of normal galaxies at the depth of AGN samples. This allows to study the relation between the space distribution of AGNs and galaxies. In the case of the CDFS, our photo- $z$ based density detects two clusters, at $z \sim 0.7$ and $z \sim 1$, already identified in the spectroscopic redshift survey of Gilli et al. (2003). A third peak at $z \sim 1.5$ in the galaxy density distribution likely corresponds to a peak which is found in the spectroscopic redshift distribution of AGNs but not of galaxies. The analysis of deeper photometric data, which is in progress (Trevese et al. 2006), is necessary to confirm the reality of this structure. Once galaxies belonging to a cluster are identified, it is possible to study the galaxy type distribution as a function of the local density. This provides an evidence of environmental effects on galaxy evolution and a way to quantify these effects. In the case of our analysis this allowed to prove, in a cluster of redshift 0.7 , that the fraction of red galaxies increases with density, as already known for lower redshift structures (Dressler 1980; and Dressler et al. 1997). A similar effect appears at redshift 1, though the evidence in this case is marginal. Clearly the density computed by our method is not the "real local density", due to the strong smoothing in the $z$ direction caused by the redshift uncertainty and to the assumptions adopted to compensate the increasing loss of faint galaxies at high redshift. Thus any cosmological application of our method, devoted to study the evolution in cosmic time of galaxy clusters properties and environmental effects, will require a calibration through a detailed comparison with simulated catalogues. Obviously the same is true for any other cluster finding technique. However, there are properties of the cluster galaxy population which, to a first approximation, do not need a comparison with mock catalogues to provide significant physical information. This is the case of the slope of the cluster red sequence which has been traced up to $z=1.27$ (Blakeslee et al. 2003, and refs. therein). According to Kodama et al. 1998 the evolution of the colour-magnitude (C-M) slope depends on relative age variations in early-type galaxies with different luminosities. Accordingly, the constancy of the slope up to redshift 1.27 is consistent with passive evolution of an old stellar population that was formed at high redshift. In this scenario, changes of the $\mathrm{C}-\mathrm{M}$ relation slope are expected at redshifts approaching the star formation phase. An alternative interpretation of the constancy of the C-M relation, in the framework of hierarchical models of galaxy formation, has been proposed by Kauffmann \& Charlot (1998). In this scenario metals, once formed, are more easily ejected from smaller disks. Large (bright) ellipticals are more metal-rich because they are formed from the mergers of large disks. In selecting rich clusters at high redshift one is biasing samples towards objects that merged at the highest redshifts and for this reason they appear to follow the passive evolution. In both scenarios the $\mathrm{C}-\mathrm{M}$ relation is due to a mass-metallicity relation and the evolution of the C-M slopes at high redshift contains critical informations on the origin of the C-M slope itself. Our analysis supports the evidence of a constant C-M slope up to $z=1$, meaning a correspondingly constant mass-metallicity relation. We stress that most of the highest redshift clusters detected so far were selected in the X-ray band, in particular those 
at $z=1.24$ (Blakeslee et al. 2003; Rosati et al. 2004) and $z=1.4$ (Mullis et al. 2005). However, both these clusters have a velocity dispersion of about $800 \mathrm{~km} \mathrm{~s}^{-1}$ and intracluster gas temperature $k T \sim 6 \mathrm{keV}$, typical of rich clusters (Bahcall 1988; Arnaud et al. 2005). The X-ray luminosities of these two clusters in the $0.5-2 \mathrm{keV}$ band are $1.9 \times 10^{44}$ and $3.0 \times 10^{44} \mathrm{erg} \mathrm{s}^{-1} h_{70}^{-2}$ respectively (Rosati et al. 2004; Mullis et al. 2005), again typical of clusters with richness class greater than 2 (Ledlow et al. 2003). Rich clusters of these redshifts are just at the limit of our present $(2+1) \mathrm{D}$ analysis based on photometric observations. Work is in progress (Trevese et al. 2006) to extend the study to a deeper samples $\left(K_{A B} \sim 27\right)$ which will allow the analysis at $z \sim 1.5$ even of poor structures of the type of those detected by photometric redshifts in the present work in the CDFS. These less pronounced structures are hardly detectable in X-rays, due to the strong dependence on richness of the X-ray luminosity (Ledlow et al. 2003), and their C-M slope could differ from that of the richest clusters, if its constancy is only apparent and mainly produced by the cluster selection bias towards richer structures.

\section{Conclusions}

We have presented a new method to detect local overdensities in the galaxy distribution, based on: i) photometric redshifts, with proper account of the relevant uncertainty, to evaluate distance; ii) distance to the $n$th neighbour to evaluate densities. From a methodological point of view, we can conclude that:

- Although our $(2+1)$ D method is limited by the redshift uncertainty, so that in principle only structures separated by more than $\delta_{\text {phot }}$ can be resolved, it still allows to dramatically increase our possibility of detecting structures respect to $2 \mathrm{D}$ analyses, as shown by the example in Fig. 9.

- If we restrict to structures of the type of Abell clusters, whose number density is about $10^{-5} \mathrm{Mpc}^{-3}$ (Bahcall 1988), the average inter-cluster distance results $d_{\mathrm{cl}} \sim 100 \mathrm{Mpc}$, namely $\delta_{\text {phot }} \leq d_{\mathrm{cl}}$, meaning that the use of photometric redshifts allows to resolve even aligned structures, as shown by the simulation in Sect. 5.

From an astrophysical point of view, our analysis of deep multiband photometry in the CDFS field allows the following conclusions:

- We have detected two structures at redshifts 0.7 and 1.0, whose existence was known from previous spectroscopic studies.

- A third structure at redshift 1.5 has also been detected but requires deeper data for confirmation.

- The fraction of red galaxies inside the structure at $z=1$ indicates a marginal density dependence while in the structure at $z=0.7$ the increase of the red fraction with density is seen very clearly; this extends the results of Dressler et al. (1997), Carlberg et al. (2001), and Tanaka et al. (2005).

- Our results add new evidence in favour of constant slope of the $\mathrm{C}-\mathrm{M}$ relation in clusters, at least up to $z=1$ implying a constant mass-metallicity relation, according to the standard interpretations.

- Our analysis shows that the average C-M relation for galaxies belonging to the red population is consistent with a linear extrapolation of the relation found by Bell et al. (2004) up to $z=1.5$, complementing the results of Giallongo et al. (2005), who have shown that the blueing of the average colour of the red galaxies extends to $z \approx 2-3$.
- The use of photometric redshifts will allow to analyse the redshift dependence of the C-M relation at high redshift, even in moderate overdensities, providing constraints on the very origin of the $\mathrm{C}-\mathrm{M}$ relation.

Thus, in spite of the low resolution in distance, $(2+1) \mathrm{D}$ analysis based on photometric redshift is an extremely valuable tool to complement other cluster finding techniques and perform large scale structure studies based on photometric surveys which, at present, posses unique capabilities in combining depth and field width.

Acknowledgements. We thank the anonymous referee for valuable comments and suggestions. We are grateful to Andrea Grazian and Sara Salimbeni for providing advise and assistance in the use of photometric and spectroscopic catalogues.

\section{References}

Abell, G. O. 1958, ApJS, 3, 211

Arnaud, M., Pointecouteau, E., \& Pratt, G. W. 2005, A\&A, 441, 893 Arimoto, N., \& Yoshii, Y. 1987, A\&A, 188, 13

Bahcall, N. A. 1988, ARA\&A, 26, 631

Balogh, M., Eke, V., Miller, C., et al. 2004, MNRAS, 348, 1355 Bell, E. F., Wolf, C., Meisenheimer, K., et al. 2004, ApJ, 608, 752 Blakeslee, J. P., Franx, M., Postman, M., et al. 2003, ApJ, 596, L143 Botzler, C. S., Snigula, J., Bender, R., \& Hopp, U. 2004, MNRAS, 349, 425 Bower, R. G., Lucey, J. R., \& Ellis, R. S. 1992, MNRAS, 254, 601 Carlberg, R. G., Yee, H. K. C., Morris, S. L., et al. 2001, ApJ, 563, 736 Cimatti, A., Daddi, E., \& Mignoli, M. 2002a, A\&A, 381, L68 Cimatti, A., Mignoli, M., Daddi, E., et al. 2002b, A\&A, 392, 395 Connolly, A. J., Csabai, I., Szalay, A. S., et al. 1995, AJ, 110, 2655 De Propris, R., Colless, M., Driver, S. P., et al. 2003, MNRAS, 342, 725 Dressler, A. 1980, AJ, 236, 351

Dressler, A., Oemler, A. Jr., Couch, W. J., et al. 1997, ApJ, 490, 577 Fontana, A., D'Odorico, S., Poli, F., et al. 2000, AJ, 120, 2206

Gal, R. R. 2006 [arXiv: astro-ph/0601195]

Giallongo, E., D’Odorico, S., Fontana, A., et al. 1998, AJ, 115, 2169 Giallongo, E., Salimbeni, S., Menci, N., et al. 2005, ApJ, 622, 116

Giacconi, R., Zirm, A., \& Wang, J. 2002, ApJS, 139, 369

Gilli, R., Cimatti, A., Daddi, E., et al. 2003, ApJ, 592, 721

Girardi, M., Giuricin, G., Mardirossian, F., Mezzetti, M., \& Boschin, W. 1998, ApJ, 505, 74

Gladders, M. D., Lopez-Cruz, O., Yee, H. K. C., \& Kodama, T. 1998, ApJ, 501, 571

Gomez, P. L., Nichol, R. C., Miller, C. J., et al. 2003, ApJ, 584, 210

Goto, T., Yagi, M., Tanaka, M., \& Okamura, S. 2004, MNRAS, 348, 515

Grazian, A., Fontana, A., De Santis, C., et al. 2006, A\&A, 449, 951

Heisler, J., Tremaine, S., \& Bahcall, J. N. 1985, ApJ, 298, 8

Hogg, D. W. 2000 [arXiv: astro-ph/9905116]

Huchra, J., \& Geller, M. 1982, ApJ, 257, 423

Kauffmann, G., \& Charlot, S. 1998, MNRAS, 294, 705

Kodama, T., Arimoto, N., Barger, A. J., \& Aragón-Salamanca, A. 1998, A\&A, 334, 99

Lanzetta, K. M., Yahil, A., \& Fernández-Soto, A. 1996, Nature, 381, 759

Ledlow, M. J., Voges, W., Owen, Frazer N., \& Burns, J. O. 2003, AJ, 126, 2740

Lumsden, S. L., Nichol, R. C., Collins, C. A., \& Guzzo, L. 1992, MNRAS, 258, 1

Menci, N., Fontana, A., Giallongo, E., et al. 2005, ApJ, 632, 49

Mullis, C. R., Rosati, P., Lamer, G., et al. 2005, ApJ, 623L, 85

Poggianti, B. M. 1997, A\&AS, 122, 399

Poli, F., Giallongo, E., Fontana, A., et al. 2003, ApJ, 593, L1

Postman, M., Lubin, L. M., Gunn, J. E., et al. 1996, AJ, 111, 615

Rosati, P., Tozzi, P., Ettori, S., et al. 2004, AJ, 127, 230

Sarazin, C. L. 1988, X-ray Emission from Clusters of Galaxies (Cambridge: Cambridge University Press)

Schechter, P. 1976, ApJ, 203, 297

Strateva, I., Ivezić, Ž., Knapp, G. R., et al. 2001, AJ, 122, 1861

Tanaka, M., Kodama, T., Arimoto, N., et al. 2005, MNRAS, 362, 268

Trevese, D., et al. 2006, in preparation

York, D. G., Adelman, J., Anderson, J. E. Jr., et al. 2000, AJ, 120, 1579

Zwicky, F., Herzog, E., \& Wild, P. 1961, Catalogue of Galaxies and of Clusters of Galaxies (Pasadena: California Institute of Technology (CIT)) 